\title{
Development of relativistic shock waves in viscous gluon matter
}

\author{
I. Bouras ${ }^{a}$, E. Molnár ${ }^{b}$, H. Niemi ${ }^{b}$, Z. Xu ${ }^{a}$, A. El $^{a}$, O. Fochler ${ }^{a}$, \\ C. Greiner ${ }^{a}$ and D.H. Rischke ${ }^{a, b}$ \\ ${ }^{a}$ Institut für Theoretische Physik, Johann Wolfgang Goethe-Universität, Max-von-Laue-Str. 1, D-60438 Frankfurt am \\ Main, Germany \\ ${ }^{b}$ Frankfurt Institute for Advanced Studies, Ruth-Moufang-Str. 1, D-60438 Frankfurt am Main, Germany
}

\begin{abstract}
To investigate the formation and the propagation of relativistic shock waves in viscous gluon matter we solve the relativistic Riemann problem using a microscopic parton cascade. We demonstrate the transition from ideal to viscous shock waves by varying the shear viscosity to entropy density ratio $\eta / s$. We show that an $\eta / s$ ratio larger than 0.2 prevents the development of well-defined shock waves on time scales typical for ultrarelativistic heavy-ion collisions. These findings are confirmed by viscous hydrodynamic calculations.
\end{abstract}

\section{Introduction}

Recently, in the context of the jet-quenching phenomena [1], exciting jet-associated particle correlations [2] have been observed in heavy-ion collisions at BNL's Relativistic Heavy-Ion Collider (RHIC). This indicates the formation of shock waves in form of Mach Cones [3] induced by supersonic partons moving through the quark-gluon plasma (QGP) and could give a direct access to the equation of state of the QGP.

Shock waves can be observed if the matter, where the shock waves are induced, behaves like a fluid. The large measured elliptic flow coefficient $v_{2}[4]$ implies that the QGP created at RHIC could be a nearly perfect fluid with a small viscosity. Calculations of viscous hydrodynamics [5] and microscopic transport theory [6] have estimated the shear viscosity to the entropy density ratio $\eta / s$ to be less than 0.4 . Still, it is not known, if this upper limit of the $\eta / s$ ratio is sufficiently small to allow the formation of relativistic shock waves.

In this work we address the question, whether and when relativistic shock waves can develop in viscous gluon matter for given $\eta / s$ values. For this purpose we consider the relativistic Riemann problem. Its initial conditions are two regions of equilibrated matter with different constant pressure separated by a membrane which is removed at $t=0$. The matter evolves in form of a one-dimensional expansion. We solve the relativistic Riemann problem employing both the parton cascade BAMPS (Boltzmann Approach of MultiParton Scatterings) [7] and the vSHASTA approach (viscous SHArp and Smooth Transport Algorithm) [8] of viscous hydrodynamics. We demonstrate the transition from ideal shock waves to free diffusion by varying the $\eta / s$ ratio from zero to infinity. We estimate the upper limit of the $\eta / s$ value, for which shocks can still be observed experimentally on the time scale typical at RHIC. 


\section{BAMPS and vSHASTA}

BAMPS is a microscopic transport model solving the Boltzmann equation

$$
p^{\mu} \partial_{\mu} f(x, p)=C(x, p)
$$

for on-shell gluons with the collision integral $C(x, p)$. The algorithm for collisions is based on the stochastic interpretation of the transition rate [7]. In this study, we consider only binary gluon scattering processes with an isotropic cross section, which is adjusted locally at each time step to keep a constant $\eta / s$ value [9, 10, 11, 12].

vSHASTA solves the Israel-Stewart (IS) equations of dissipative hydrodynamics. In $1+1$ dimensions IS equations reduce to [9]

$$
\begin{aligned}
& \partial_{t} T^{00}+\partial_{z}\left(v T^{00}\right)=-\partial_{z}(v P+v \tilde{\pi}), \\
& \partial_{t} T^{0 z}+\partial_{z}\left(v T^{0 z}\right)=-\partial_{z}(P+\tilde{\pi}), \\
& \gamma \partial_{t} \tilde{\pi}+\gamma v \partial_{z} \tilde{\pi}=\frac{1}{\tau_{\pi}}\left(\pi_{N S}-\tilde{\pi}\right)-\frac{\tilde{\pi}}{2}\left(\theta+D \ln \frac{\beta_{2}}{T}\right),
\end{aligned}
$$

where $\theta \equiv \partial_{\mu} u^{\mu}$ and $D \equiv u^{\mu} \partial_{\mu}$. For vanishing shear viscous pressure, $\tilde{\pi}$, Eqs. (2) and (3) reduce to the equations of ideal hydrodynamics.

\section{Development of shock waves}

Figure 1 shows the solution of the relativistic Riemann problem for various $\eta / s$ values as computed with BAMPS. The results demonstrate a gradual transition from ideal hydrodynamic
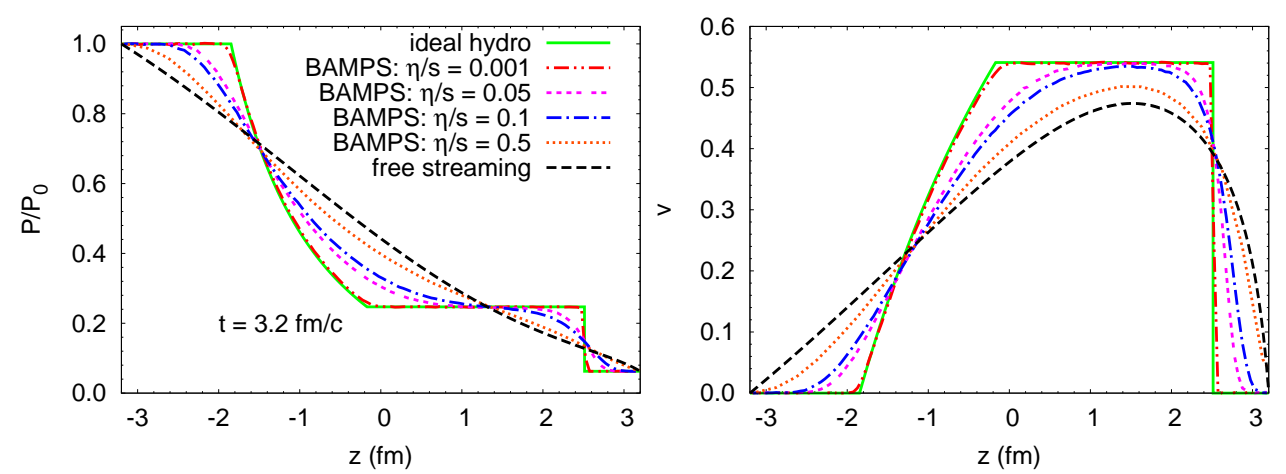

Figure 1: (Color online) The solution of the Riemann problem. At $t=0$, the pressure is $P_{0}=5.43 \mathrm{GeVfm}^{-3}$ for $z<0$ and $P_{4}=0.33 \mathrm{GeVfm}^{-3}$ for $z>0$. The left panel shows the pressure and the right panel the velocity at time $t=3.2 \mathrm{fm} / \mathrm{c}$.

limit $(\eta=0)$ to free streaming of particles $(\eta=\infty)$. The ideal solution [13] is reproduced with a very good precision for $\eta / s=0.001$. We see that a shock wave is traveling into matter with lower pressure (on the right) with a velocity $v_{\text {shock }}$ larger than the velocity of sound $c_{s}$. The region behind the shock front is the so-called shock plateau, where matter is equilibrated and moves collectively with $v_{\text {plat }}$, shown on the right panel of Fig. 1. Simultaneously a rarefaction wave is propagating with $c_{s}$ into matter of higher pressure (on the left). 
A larger $\eta / s$ value results in a finite transition layer where the quantities change smoothly rather than discontinuously as in the case of a perfect fluid. Furthermore a non-zero viscosity, if large enough, impedes the formation of a shock plateau and a clear separation of the shock front from the rarefaction fan.
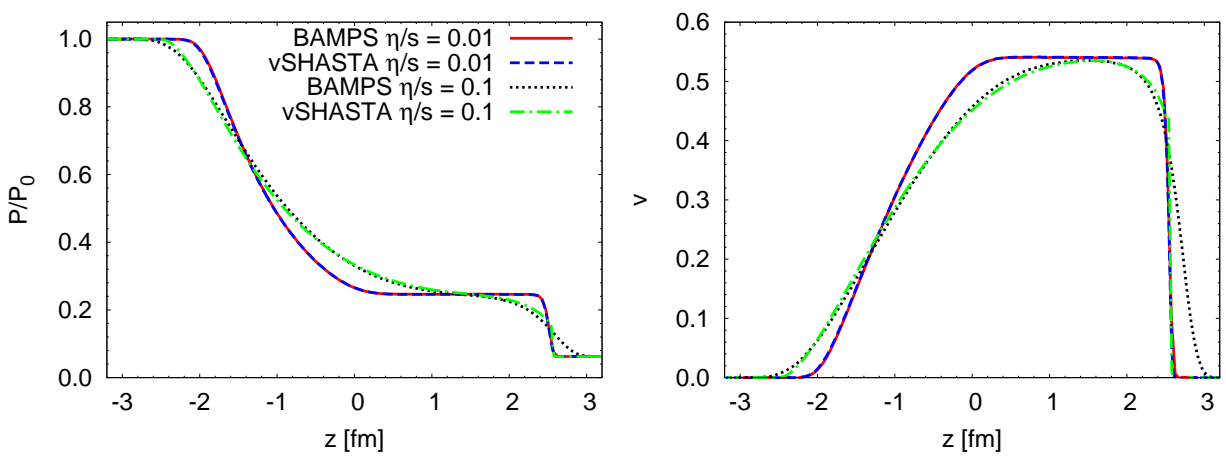

Figure 2: (Color online) Same as in Fig. 1 Results are obtained using BAMPS and vSHASTA.

In Fig. 2 we compare the results from BAMPS and vSHASTA for $\eta / s=0.01$ and 0.1. We see a perfect agreement for $\eta / s=0.01$, whereas for larger value of $\eta / s=0.1$ small deviations in the region of the shock front and rarefaction wave are found. The reason for the difference is that in these regions the local Knudsen number $K_{\theta}=\lambda_{\operatorname{mfp}} \partial_{\mu} u^{\mu}$ [14] is large and thus the applicability of IS equations is questionable. Transport calculations do not suffer from that drawback.

\section{Time scale of formation of shock waves}
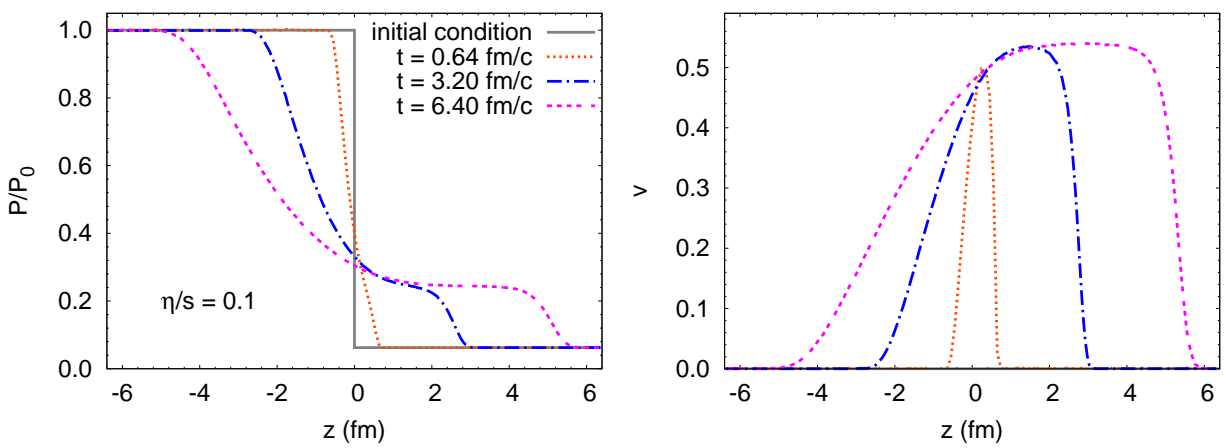

Figure 3: (Color online) Same as in Fig. 1 Results are obtained using BAMPS for $\eta / s=0.1$.

The formation of a shock wave takes a certain amount of time, as demonstrated in Fig. 3 for $\eta / s=0.1$. At early times a shock has not yet developed, the profile looks like a free streaming of particles. But at later times we observe the creation of a shock plateau. Formally we define the time of formation of the shock plateau when the maximum of the velocity distribution $v(z)$ reaches the value $v_{\text {plat }}$ of the ideal-fluid solution in Fig. 1. From the right panel of Fig. 3, we see that this happens at $t=3.2 \mathrm{fm} / \mathrm{c}$. 
In Ref. [9] we have found the scaling behavior of the Riemann problem: the time scale of the formation of shock waves $t_{f}$ is proportional to the $\eta / s$ ratio. From Fig. 3 we also infer that, for $\eta / s>0.1$, a shock plateau has not yet developed at $t=3.2 \mathrm{fm} / \mathrm{c}$, whereas for $\eta / s<0.1$, it has already fully formed. For $\eta / s>0.2$ we obtain $t_{f}>6.4 \mathrm{fm} / \mathrm{c}$, which is the typical lifetime of the QGP produced in heavy-ion collisions at RHIC. Therefore, an imperfect quark gluon fluid with $\eta / s>0.2$ will prevent the observation of Mach Cone signals at RHIC.

\section{Conclusion}

Using the parton cascade BAMPS we have solved the relativistic Riemann problem. The transition from ideal-fluid behavior to free streaming is demonstrated. Numerical results from BAMPS agree well with those obtained from viscous hydrodynamical calculations using vSHASTA. Considering the scaling behavior we found that the formation of shock waves in gluon matter with $\eta / s>0.2$ probably takes longer than the lifetime of the QGP at RHIC.

\section{Acknowledgments}

This work was supported by the Helmholtz International Center for FAIR within the framework of the LOEWE program launched by the State of Hesse. E. Molnár acknowledges support by the Alexander von Humboldt foundation. The work of $\mathrm{H}$. Niemi was supported by the Extreme Matter Institute (EMMI).

\section{References}

[1] J. Adams et al. [STAR Collaboration], Phys. Rev. Lett. 91, 172302 (2003); A. Adare et al. [PHENIX Collaboration], ibid. 101, 232301 (2008).

[2] F. Wang [STAR Collaboration], J. Phys. G 30, S1299 (2004); J. Adams et al. [STAR Collaboration], Phys. Rev. Lett. 95, 152301 (2005); S. S. Adler et al. [PHENIX Collaboration], ibid. 97, 052301 (2006); J. G. Ulery [STAR Collaboration], Nucl. Phys. A 774, 581 (2006); N. N. Ajitanand [PHENIX Collaboration], ibid. 783, 519 (2007); A. Adare et al. [PHENIX Collaboration], Phys. Rev. C 78, 014901 (2008).

[3] H. Stöcker, Nucl. Phys. A 750, 121 (2005); J. Ruppert and B. Müller, Phys. Lett. B 618, 123 (2005); J. CasalderreySolana, E. V. Shuryak and D. Teaney, J. Phys. Conf. Ser. 27, 22 (2005); V. Koch, A. Majumder and X. N. Wang, Phys. Rev. Lett. 96, 172302 (2006).

[4] S. S. Adler et al. [PHENIX Collaboration], Phys. Rev. Lett. 91, 182301 (2003); J. Adams et al. [STAR Collaboration], ibid. 92, 052302 (2004); B. B. Back et al. [PHOBOS Collaboration], Phys. Rev. C 72, 051901 (2005).

[5] M. Luzum and P. Romatschke, Phys. Rev. C 78, 034915 (2008); H. Song and U. W. Heinz, arXiv:0812.4274

[6] Z. Xu, C. Greiner and H. Stöcker, Phys. Rev. Lett. 101, 082302 (2008); Z. Xu and C. Greiner, Phys. Rev. C 79, 014904 (2009).

[7] Z. Xu and C. Greiner, Phys. Rev. C 71, 064901 (2005); 76, 024911 (2007).

[8] E. Molnar, H. Niemi and D. H. Rischke, arXiv:0907.2583 [nucl-th]. E. Molnar, Eur. Phys. J. C 60 (2009) 413

[9] I. Bouras et al., Phys. Rev. Lett. 103, 032301 (2009)

[10] Z. Xu and C. Greiner, Phys. Rev. Lett. 100, 172301 (2008).

[11] P. Huovinen and D. Molnar, Phys. Rev. C 79, 014906 (2009).

[12] A. El et al., Phys. Rev. C 79, 044914 (2009)

[13] V. Schneider et al., J. Comput. Phys. 105, 92 (1993); D. H. Rischke, S. Bernard and J. A. Maruhn, Nucl. Phys. A 595, 346 (1995); D. H. Rischke, arXiv:nucl-th/9809044

[14] B. Betz, D. Henkel and D. H. Rischke, arXiv:0812.1440 